\title{
From Prochiral N-Heterocyclic Carbenes (NHC) to Optically Pure Metal Complexes: New Opportunities in Asymmetric Catalysis
}

Lingyu Kong,,${ }^{\S}$ Jennifer Morvan, ${ }^{\dagger}$ Delphine Pichon, ${ }^{\dagger}$ Marion Jean, ${ }^{\S}$ Muriel Albalat,${ }^{\S}$ Thomas Vives, ${ }^{\dagger}$ Sophie Colombel-Rouen, ${ }^{\dagger}$ Michel Giorgi, ${ }^{\perp}$ Vincent Dorcet,${ }^{\dagger}$ Thierry Roisnel,${ }^{\dagger}$ Christophe Crévisy, ${ }^{\dagger}$ Didier Nuel, ${ }^{\S}$ Paola Nava, ${ }^{\S}$ Stéphane Humbel, ${ }^{\S}$ Nicolas Vanthuyne, ${ }^{\S}$ Marc Mauduit ${ }^{*, \dagger}$ and Hervé Clavier*,§

\author{
$\S$ Aix Marseille Univ, CNRS, Centrale Marseille, iSm2, Marseille, France \\ † Univ Rennes, Ecole Nationale Supérieure de Chimie de Rennes, CNRS, ISCR UMR 6226, F-35000 Rennes, France \\ $\perp$ Aix Marseille Univ, CNRS, Centrale Marseille, FSCM, Marseille, France
}

\section{Supporting Information Placeholder}

\begin{abstract}
Well-defined optically pure Transition Metal-complexes bearing $C_{1}$ - and $C_{2}$-symmetric $N$ - Heterocyclic Carbene (NHC) ligands were prepared from prochiral NHC precursors. As predicted by DFT calculations, our strategy capitalizes on the formation of a metal-carbene bond which induces an axis of chirality. Configurationally stable atropisomers of various $\mathrm{NHC}$ containing TM-complexes were isolated by preparative HPLC on a chiral stationary phase in good yields and excellent optical purities (up to $99.5 \%$ ee). The carbene transfer from an optically pure $\mathrm{Cu}$-complex to gold or palladium center reveals, for the first time, a full stereoretentivity, supporting the hypothesis of an associative mechanism for the transmetalation. The potential of these new chiral TM-complexes was illustrated in asymmetric catalysis with up to $98 \%$ ee.
\end{abstract}

Due to their unique topology along with a highly modular steric environment around the metal, chiral $\mathrm{N}$ heterocyclic carbenes (NHCs) rapidly emerged as stereo-directing ligands. ${ }^{1}$ Since the first report of a highly enantioselective reaction in $2001,{ }^{2}$ chiral NHCs were intensively studied in enantioselective catalysis with resounding breakthroughs. ${ }^{3}$ Advantageously, their versatile and easy synthetic access led to the development of a plethora of chiral NHCs containing various elements of symmetry. Among them, chiral Transition Metal (TM) complexes containing $C_{2}$ - or $C_{1}$-symmetric NHC precursors 1 and $\mathbf{2}$ proved to be quite efficient, thanks to the effective chiral relay from the stereogenic substituents of carbene backbone to the $\mathrm{N}$-aryl ortho-substituent that induces a trans-relationship (Figure 1,a). ${ }^{4}$ The resulting chiral environment close to the metal enabled to reach remarkable stereo-inductions (up to $>99 \%$ ee) in numerous asymmetric catalytic transformations. ${ }^{3}$ Despite these notable achievements, the technology remains somewhat costly as optically pure starting materials are required (in both enantiomers if possible) for the synthesis of NHCs.
Consequently, reducing the cost of chiral technology remains a longstanding goal for chemists. Moreover, the chiral relay generates a mismatch effect which might be detrimental to chiral inductions. ${ }^{4}$ We report herein the synthesis of many optically pure ( $>98 \%$ ee) well-defined $C_{1}$ - and $C_{2}$-symmetric NHC-TM complexes containing an axial chirality, which may be readily synthesized from prochiral NHC precursors (Figure 1,b). ${ }^{5}$ The chiral resolution of resulting stable atropisomers is efficiently achieved by HPLC on a preparative scale. ${ }^{6}$

(a) The chiral relay strategy for $C_{1}$ - and $C_{2}$-symmetric chiral NHC precursors
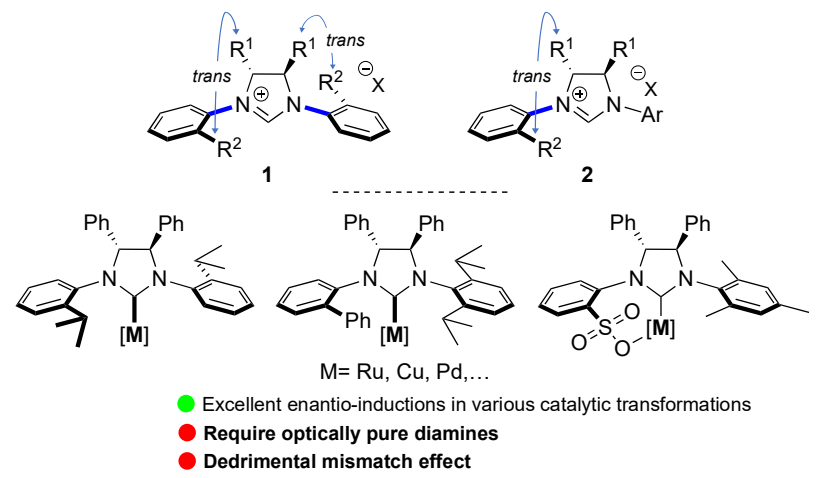

(b) New access to chiral TM-complexes from prochiral NHCs (This work) :

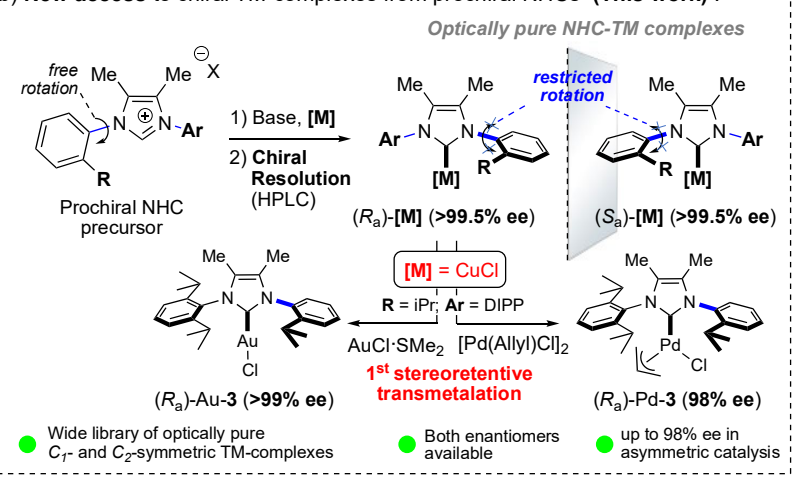

Figure 1. Design of chiral NHC-TM catalysts 
These new $C_{1-}$ and $C_{2}$-symmetric chiral $\mathrm{Cu}$ - and $\mathrm{Pd}$ complexes display excellent performances in asymmetric catalysis (up to $98 \%$ ee). Importantly, and for the first time, we demonstrate that the carbene transfer from an optically pure $\mathrm{Cu}$-complex to gold or palladium center occurred with a full stereoretentivity giving experimental insights to the NHC transmetalation mechanism.

Our study began with the design of prochiral $\mathrm{NHC}$ precursors, i.e. imidazolium salts $\mathbf{3} \cdot \mathrm{Cl}$ (Figure 2). Given the infinite substitution patterns that could be considered either on the carbene backbone or on $N$-aryl substituents, prior theoretical calculations appeared useful to determine with accuracy the expected rotational barriers of the $N$-aryl bond after coordination to copper(I) chloride $\left(>93 \mathrm{~kJ} \cdot \mathrm{mol}^{-1} ; t_{1 / 2}>1000 \mathrm{~s}\right.$ at $25{ }^{\circ} \mathrm{C}$ to observe atropisomers, but ideally $>110 \mathrm{~kJ} \cdot \mathrm{mol}^{-1} ; t_{1 / 2}>12$ days at $\left.25^{\circ} \mathrm{C}\right) .^{7}$ With a bulky isopropyl group on ortho position of $N$-aryl substituents $(\mathrm{Cu}-\mathbf{3 a})$, the rotation barrier values are too low to obtain stable atropisomers (Figure 2,a; $\Delta \mathrm{G}^{\neq}(\mathrm{Cu})=$ $51.0 \mathrm{~kJ} \cdot \mathrm{mol}^{-1}$ and $\left.\Delta \mathrm{G}^{\neq}(\mathrm{BB})=42.6 \mathrm{~kJ} \cdot \mathrm{mol}^{-1}\right){ }^{8}$

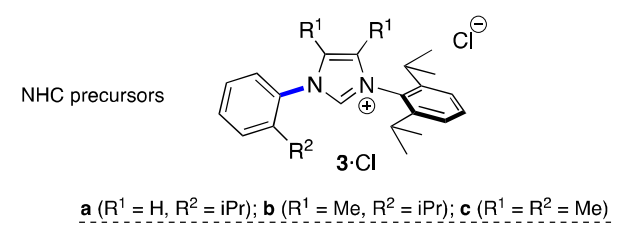

(a) Rotamers (free rotations on copper and backbone sides)

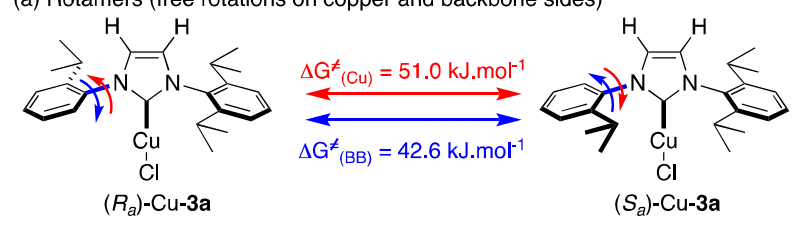

(b) Atropisomers (restricted rotations)

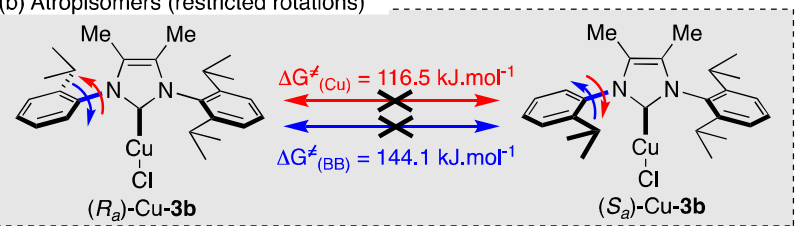

(c) Rotamers (free rotation on copper side)

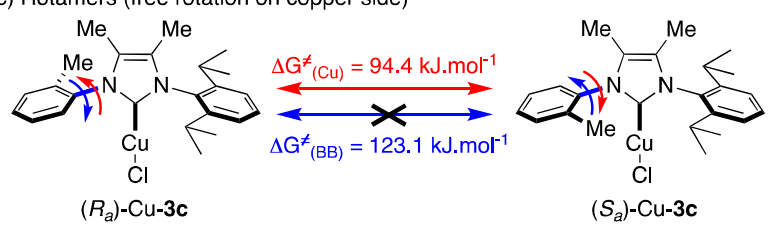

Figure 2. DFT calculations of rotational barriers for NHCcopper complexes $\mathrm{Cu}-\mathbf{3 a},-\mathbf{3 b},-\mathbf{3 c} . \Delta \mathrm{G}^{\neq}(\mathrm{Cu})$ and $\Delta \mathrm{G}^{\neq}(\mathrm{BB})$ are the values of rotation barriers on backbone (BB) and copper $(\mathrm{Cu})$ sides, respectively.

Nevertheless, the backbone substitution with methyl groups $(\mathrm{Cu}-\mathbf{3 b})$ leads to a substantial increase of the rotation barriers values up to expect configurationally stable enantiomers (Figure 2,b, $\Delta \mathrm{G}^{\neq}{ }_{(\mathrm{Cu})}=116.4 \mathrm{~kJ} \cdot \mathrm{mol}^{-1}$ and $\left.\Delta \mathrm{G}_{(\mathrm{BB})}^{\neq}=144.1 \mathrm{~kJ} \cdot \mathrm{mol}^{-1}\right)$. Of note, despite the methyl groups on the backbone, a methyl substituent in ortho position of the aryl group $(\mathrm{Cu}-3 \mathrm{c})$ could not prevent the aryl rotation (Figure 2,c, $\Delta \mathrm{G}^{\neq}(\mathrm{Cu})=94.4 \mathrm{~kJ} \cdot \mathrm{mol}^{-1}$ and $\left.\Delta \mathrm{G}_{(\mathrm{BB})}^{\neq}=123.1 \mathrm{~kJ} \cdot \mathrm{mol}^{-1}\right)$. In order to assess experimentally these data obtained from theoretical calculations, $\mathrm{Cu}-\mathbf{3 b}$ complex was synthesized from the prochiral imidazolium salt $\mathbf{3 b} \cdot \mathbf{C l}$ (Scheme 1, see Supporting Information; SI). ${ }^{9}$ The deprotonation of the latter by $\mathrm{K}_{2} \mathrm{CO}_{3}$ in the presence of $\mathrm{CuCl}$ afforded the desired complex $\mathrm{Cu}-\mathbf{3 b}$ in $57 \%$ yield after silica gel purification. ${ }^{10}$ The HPLC analysis on chiral stationary phase confirmed clearly that stable atropisomers were formed in a racemic mixture (Scheme 1).

Scheme 1. Synthesis of optically pure copper-complexes $\mathrm{Cu}-3 \mathrm{~b}$ from prochiral imidazolium $\mathbf{3 b} \cdot \mathrm{Cl}$

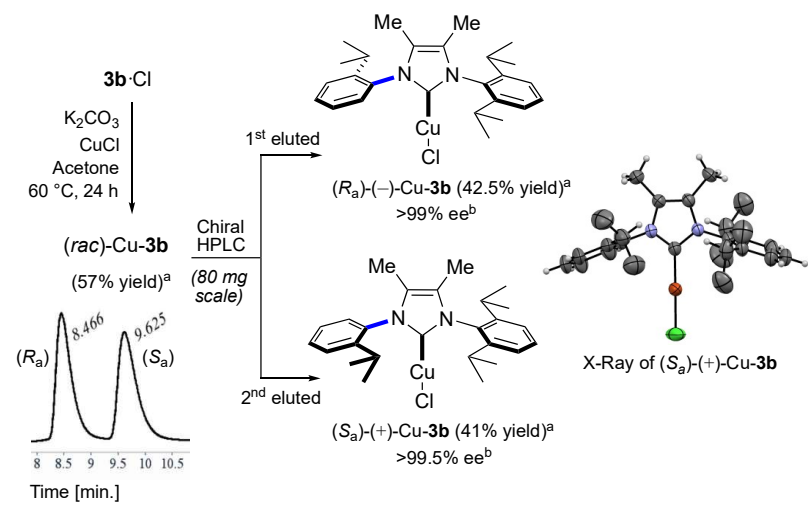

${ }^{a}$ Isolated yields. ${ }^{b}$ Determined by chiral-stationary phase HPLC analysis.

Thanks to the robustness of copper-NHC complexes toward silica gel, the chiral resolution of $(\mathrm{rac})-\mathrm{Cu}-3 \mathbf{b}$ by HPLC on a preparative scale $(80 \mathrm{mg}$, flow-rate $=5$ mL.min. ${ }^{-1}$; see SI) enabled to isolate both atropisomers $(+)-\mathrm{Cu}-3 \mathbf{b}$ and (-)-Cu-3b in excellent yields and remarkable $>99 \%$ optical purities. Moreover, the Electronic Circular Dichroism (ECD), affording chiroptical properties of the copper complex, showed expected mirror-image spectra for both enantiomers (see SI, Figure S4). X-ray diffraction analysis of the second eluted atropisomers $(+)-\mathrm{Cu}-\mathbf{3 b}$ confirmed its structure but also enabled to determine its absolute configuration $\left(S_{\mathrm{a}}\right.$, Scheme 1). Furthermore, kinetic of enantiomerization of $\left(S_{\mathrm{a}}\right)-(+)-\mathrm{Cu}-3 \mathrm{~b}$ in 1,2-dichloroethane at $83.5{ }^{\circ} \mathrm{C}$ gave access to the experimental rotation barrier value $\left(\Delta \mathrm{G}^{\neq}=\right.$ $\left.117.2 \mathrm{~kJ} \cdot \mathrm{mol}^{-1}\right)$ which fits with the predicted lowest value $\left(\Delta \mathrm{G}^{\neq}(\mathrm{Cu})=116.5 \mathrm{~kJ} \cdot \mathrm{mol}^{-1}\right.$, see Figure $\left.2, \mathrm{~b}\right)$. This validates the use of theoretical calculations as a reliable tool to design the NHC structures.

We next turned our attention to the synthesis of other optically pure atropisomeric NHC transition metal complexes. On this concern, the transmetalation represents a fundamental organometallic reaction as numerous transition-metal complexes were and are synthesized via this process. ${ }^{11}$ Furthermore, the elucidation of its mechanism, notably when coinage NHC-TM complexes are involved, remains a longstanding goal for organometallic chemists. The stable optically pure atropisomers of copper-NHC complexes, in which the axis of chirality is induced by the metal-carbene bond, represents an oppor- 
tunity to generate other TM-complexes and to gain valuable insights about the mechanistic route of transmetalation (vide infra). ${ }^{12}$ In that respect, the optically pure $\left(S_{\mathrm{a}}\right)-(+)-\mathrm{Cu}-3 \mathbf{b}$ complex $(>99.5 \%$ ee) was treated with $\mathrm{AuCl} \cdot \mathrm{SMe}_{2}$ complex in dichloromethane at $40{ }^{\circ} \mathrm{C}$ over 2 $\mathrm{h}$ (Scheme 2, a). ${ }^{13}$ To our delight, the corresponding gold complex Au-3b was isolated in quantitative yield. HPLC analysis confirmed the high enantiopurity of the newly formed gold complex ( $>99.5 \%$ ee) attesting that no racemization occurred during the transmetalation.

Scheme 2. Stereoretentive transmetalation affording optically pure gold and palladium complexes

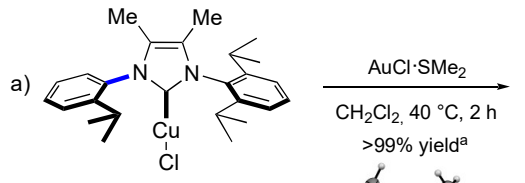

$\left(S_{a}\right)-(+)-C u-3 b>99.5 \% e^{a}$

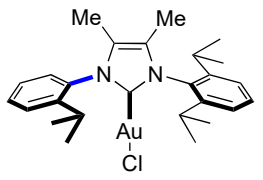

$\left(S_{\mathrm{a}}\right)-(+)-A u-3 b \quad>99.5 \% \mathrm{ee}^{\mathrm{a}}$

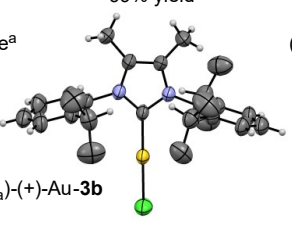

X-ray of $\left(S_{\mathrm{a}}\right)-(+)-$ Au-3b

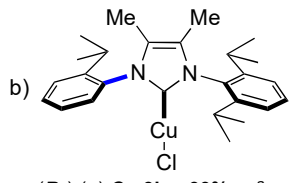

$>99 \%$ yield $^{a}$

$\left(R_{\mathrm{a}}\right)-(-)-\mathrm{Cu}-3 \mathrm{~b}>99 \% \mathrm{ee}^{\mathrm{a}}$

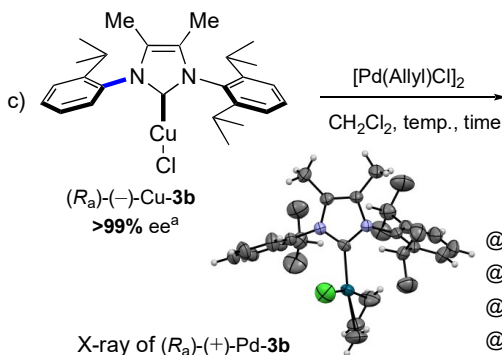

X-ray of $\left(R_{\mathrm{a}}\right)-(+)-\mathrm{Pd}-3 \mathrm{~b}$

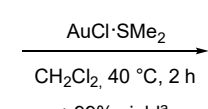

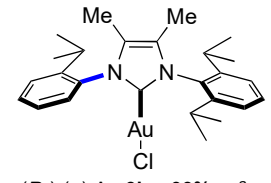

$\left(R_{\mathrm{a}}\right)-(-)-\mathrm{Au}-3 \mathrm{~b}>99 \% \mathrm{ee}^{\mathrm{a}}$

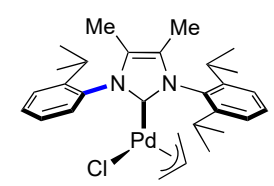

$\left(R_{\mathrm{a}}\right)-(+)-\mathrm{Pd}-3 \mathrm{~b}$

$20{ }^{\circ} \mathrm{C}, 24 \mathrm{~h}: 86 \%$ yield ${ }^{\mathrm{a}}, \mathbf{9 8 \%} \mathrm{ee}^{\mathrm{a}}$ @ $40{ }^{\circ} \mathrm{C}, 20 \mathrm{~h}: 83 \%$ yield ${ }^{\mathrm{a}}, 95 \%$ ee $\mathrm{e}^{\mathrm{a}}$ @ $60{ }^{\circ} \mathrm{C}^{\mathrm{b}}, 5 \mathrm{~h}: 86 \%$ yield $^{\mathrm{a}}, 92 \% \mathrm{ee}^{\mathrm{a}}$ @ $80^{\circ} \mathrm{C}^{\mathrm{b}}, 2 \mathrm{~h}: 86 \%$ yield $^{\mathrm{a}}, 81 \% \mathrm{ee}^{\mathrm{a}}$
${ }^{a}$ Determined by chiral-stationary phase HPLC analysis. ${ }^{b}$ Reaction performed in dichloroethane.

Moreover, X-ray diffraction analysis allowed us to confirm the complex structure and determine its absolute configuration ( $S_{\mathrm{a}}$, Scheme 2$)$. Similarly, the transmetalation starting from $\left(R_{\mathrm{a}}\right)-(-)-\mathrm{Cu}-3 \mathbf{b}$ afforded the corresponding gold enantiomer $\left(R_{\mathrm{a}}\right)-(-)$-Au-3b in quantitative yield and full optical purity, indicating unambiguously the stereoretentivity of the transmetalation (Scheme 2, b). Importantly the rotation barriers of $\mathrm{Au}-\mathbf{3 b}$ were assessed both by DFT calculations $\left(\Delta \mathrm{G}^{\neq}(\mathrm{Au})=145.5\right.$ $\mathrm{kJ} \cdot \mathrm{mol}^{-1}$ and $\left.\Delta \mathrm{G}^{\neq}(\mathrm{BB})=158.5 \mathrm{~kJ} \cdot \mathrm{mol}^{-1}\right)$ and experimentally $\left(\Delta \mathrm{G}^{\neq}=142.4 \mathrm{~kJ} \cdot \mathrm{mol}^{-1}\right.$ at $132{ }^{\circ} \mathrm{C}$ in chlorobenzene $)$, showing their enhancement over the analogous $\mathrm{Cu}-$ complex. The transmetalation process was then successfully extended to $\pi$-allyl palladium chloride (Scheme 2, c). ${ }^{13}$ Nevertheless, a prolonged reaction time $(24 \mathrm{~h})$ was required to reach a good $86 \%$ isolated yield. The optical purity of the newly formed Pd-complex (98\% ee) was confirmed by HPLC analysis attesting again that no racemization occurred during the transmetalation. In order to shorten the reaction time, the media was heated up to $80{ }^{\circ} \mathrm{C}$. Satisfactory, similar isolated yields could be reached with a duration dropping to $2 \mathrm{~h}$. However, a slight erosion of the optical purity was observed from $95 \%$ ee at $40{ }^{\circ} \mathrm{C}$ to $81 \%$ ee at $80{ }^{\circ} \mathrm{C}$. This behavior could be relied to a racemization of the starting copper complex occurring at this temperature. ${ }^{14}$ Indeed, the rotation barriers of $\mathrm{Pd}-\mathbf{3 b}$ obtained from DFT calculations $\left(\Delta \mathrm{G}^{\neq}(\mathrm{Pd})=133.4 \mathrm{~kJ} \cdot \mathrm{mol}^{-1}\right.$ and $\left.\Delta \mathrm{G}_{(\mathrm{BB})}^{\neq}=159.4 \mathrm{~kJ} \cdot \mathrm{mol}^{-1}\right)$ and experimentally $\left(\Delta \mathrm{G}^{\neq}=131.5 \mathrm{~kJ} \cdot \mathrm{mol}^{-1}\right.$ at $132{ }^{\circ} \mathrm{C}$ in chlorobenzene) are higher than for $\mathrm{Cu}-3 \mathbf{b}$. $\left(R_{\mathrm{a}}\right)-(-)-\mathrm{Cu}-$ 3b is quite stable at $40{ }^{\circ} \mathrm{C}$ with a half-life time of 22 days, but at higher temperatures, the enantiomerization occurs rapidly $\left(t_{1 / 2}=3 \mathrm{~h}\right.$ at $\left.80^{\circ} \mathrm{C}\right)$. Regarding palladium and gold counterparts, they show greatly higher stabilities, even at $80{ }^{\circ} \mathrm{C}$, with half-life times up to 15 days and 2 years respectively. ${ }^{14}$ Considering the aforementioned experimental results, a hypothetic reaction pathway for the transmetalation process is depicted in Figure 4. First, the observed stereoretentivity supports that an associative pathway could occur for the transmetalation of coinage NHC-TM complexes (Figure 3, A).

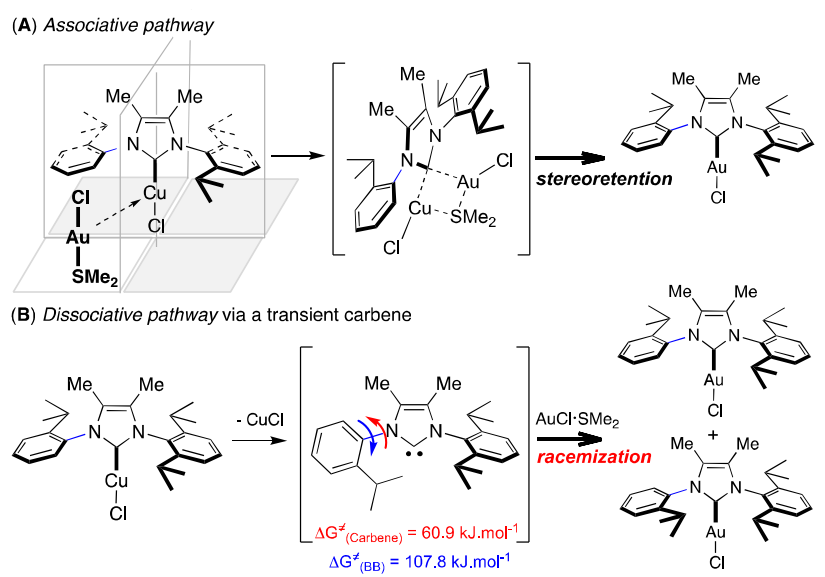

Figure 3. Proposed associative (A) vs dissociative (B) transmetalation pathway.

Indeed, a dissociative pathway (Figure 3, B) that would involve a transient free carbene seems incompatible with stereoretention as partial to full racemization could happen due to the free rotation around the $N$-Aryl bond, ${ }^{15}$ as demonstrated by DFT calculations with low values for rotational barriers on free $\mathrm{NHC}\left(\Delta \mathrm{G}^{\neq}{ }_{\text {(Carbene) }}=60.9\right.$ $\mathrm{kJ} \cdot \mathrm{mol}^{-1} ; \mathrm{t}_{1 / 2}=0.8 \mathrm{~ms}$ at $\left.40^{\circ} \mathrm{C}\right)$. Second, considering the steric hindrance within the metal coordination sphere, a four-center transition state is suspected for the associative pathway, probably in the less sterically hindered pocket in opposite side to that of $i$ Pr-aryl substituents (Figure 3, A). DFT calculations related to the formation of $\mathrm{Au}-\mathbf{3 b}$ from $\mathrm{Cu}-\mathbf{3 b}$ are currently underway to provide useful information regarding the transmetalation pathway. Of note, Au-3b and Pd-3b could be also prepared as heterochiral complexes from the corresponding imidazolium and then efficiently separated by chiral HPLC on preparative scale. 
Following the aforementioned protocol depicted in scheme 1, various optically pure $C_{1}$-symmetric $\mathrm{Cu}$ complexes were synthesized from NHC precursors $\mathbf{3 b}$,dh featuring bulky ortho-substituents (Scheme 3). In all cases, stable atropisomeric complexes were formed and each enantiomer was isolated in moderate to excellent yields and high optical purities. Moreover, the absolute configuration of each complex was assigned via X-ray diffraction analysis. ${ }^{16}$ The methodology was then extended to synthesis of $C_{2}$-symmetric NHC complexes from symmetric NHC precursors 4a-c (Scheme 4). Nevertheless, the formation of meso complexes could complicate the cHPLC resolution in addition to lower the quantity of expected chiral complexes. Indeed, with imidazolium $\mathbf{4 a} \cdot \mathrm{Cl}$ and $\mathbf{4 a} \cdot \mathrm{BF}_{4}$, meso and heterochiral complexes $\mathrm{Cu}-\mathbf{4 a}$ and $\mathrm{Pd}-\mathbf{4 a}$ were respectively isolated with ratios of 1:1.8 and 2.2:1 (Scheme 4). Fortunately, the preparative cHPLC allowed their efficient separations and the expected $C_{2}$-symmetric homochiral Pd-4a and $\mathrm{Cu}-\mathbf{4 a}$ complexes were isolated in moderate to good yields $(27-34 \%)$ and remarkable optical purities ( $>99.5 \%$ ee), highlighting the versatility of the concept. Heterochiral salts could easily be separated from the meso stereoisomers and used to prepare complexes Pd$\mathbf{4 b}$ and $\mathrm{Pd}-\mathbf{4 c}$, which were isolated free of meso isomers, and very efficiently resolved on preparative cHPLC. For instance, both enantiomers of Pd-4b were separated with $91 \%$ yield on $580 \mathrm{mg}$-scale in only 3 hours. DFT calculations confirmed that an additional ortho-substituent, even a fluorine, is sufficient to restrict the aryl rotation along the $\mathrm{N}-\mathrm{C}$ bond which leads to configurationally stable NHC precursors (lowest rotation barriers: for 4a.BF4: $\Delta \mathrm{G}_{(\mathrm{H})}^{\neq}=69.7 \mathrm{~kJ} \cdot \mathrm{mol}^{-1}$; for $\mathbf{4 b} \cdot \mathrm{BF}_{4}: \Delta \mathrm{G}_{(\mathrm{H})}^{\neq}=$ $155.9 \mathrm{~kJ} \cdot \mathrm{mol}^{-1}$; for $\left.\mathbf{4 c} \cdot \mathrm{BF}_{4}: \Delta \mathrm{G}_{(\mathrm{H})}^{\neq}=108.7 \mathrm{~kJ} \cdot \mathrm{mol}^{-1}\right) .{ }^{17}$

Scheme 3. Library of optically pure $C_{1}$-symmetric NHC-copper complexes

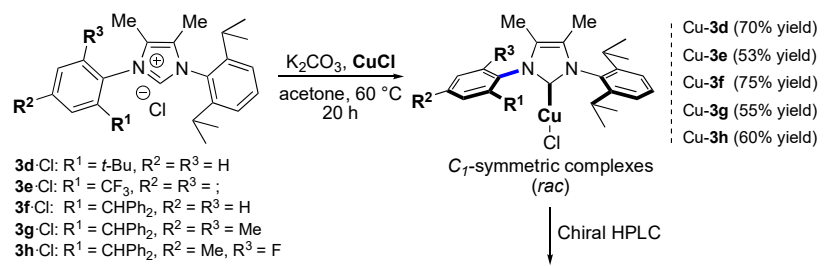

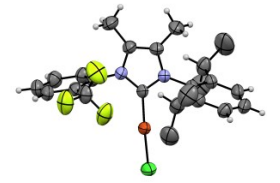

X-ray of $\left(S_{\mathrm{a}}\right)-(-)-\mathrm{Cu}-3 \mathrm{e}$

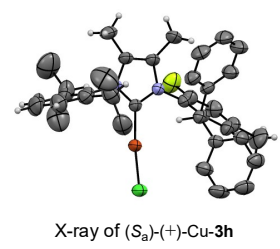

$\left(S^{2}\right)(+)-C_{-3 h}$

${ }^{a}$ Isolated yields after preparative chiral resolution. ${ }^{b}$ Determined by chiralstationary phase HPLC analysis. $\stackrel{\mathrm{Cu}}{\mathrm{Cl}}$
Scheme 4. Library of optically pure $C_{2}$-symmetric NHC-copper and -palladium complexes

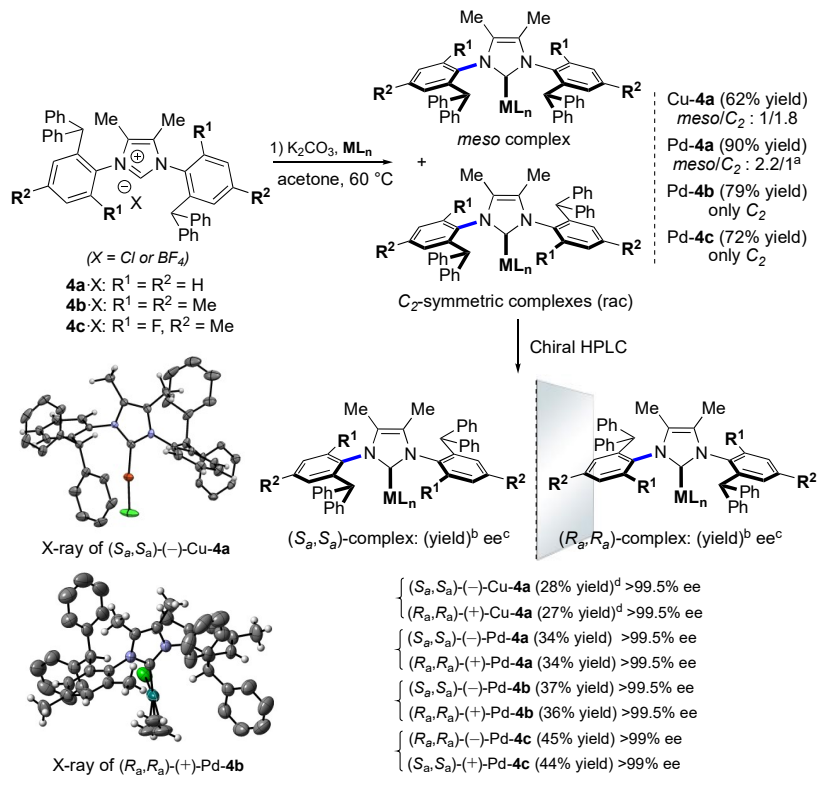

${ }^{a}$ Diastereomers were separated by $\mathrm{SiO}_{2}$ chromatography. ${ }^{b}$ Isolated yields after preparative chiral resolution. ${ }^{c} 34 \%$ of meso $\mathrm{Cu}-4$ a was also isolated. ${ }^{d}$ Determined by cHPLC analysis.

X-ray diffraction analysis of homochiral complexes confirmed their structure and enabled to determine their related absolute configurations (Scheme 4). ${ }^{16}$ Having these new $C_{1}$ - and $C_{2}$-symmetric NHC TM-complexes in hands, we next investigated their catalytic performances in asymmetric catalysis. First, the copper-catalyzed asymmetric allylic alkylation ${ }^{18}$ of diethylzinc to allyl phosphates 8 was explored (Scheme 5,a).

Scheme 5. Catalytic performances of optically pure NHC Cu- and Pd-complexes in asymmetric catalysis a) Cu-catalyzed Asymmetric Allylic Alkylation (AAA)

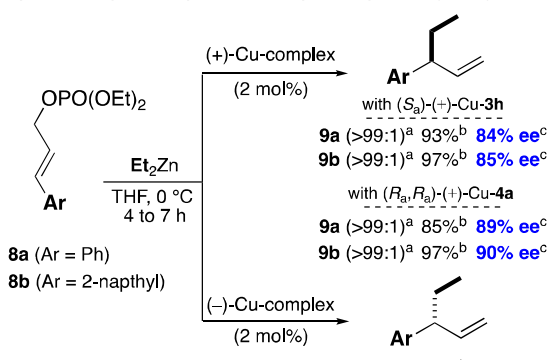

with $\left(R_{\mathrm{a}}\right)-(-)-\mathrm{Cu}-3 \mathrm{~h}:(e n t)-9 \mathrm{~b}(>99: 1)^{\mathrm{a}} 92 \%$ yield $\mathrm{d}^{\mathrm{b}} 84 \% \mathrm{ee}^{\mathrm{c}}$

with $\left(S_{a}, S_{\mathrm{a}}\right)-(-)-C u-4 a:(e n t)-9 b(>99: 1)^{a} 94 \%$ yield ${ }^{b} 89 \% e^{c}$

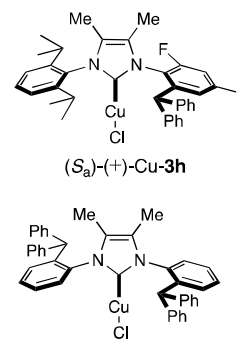

$\left(R_{\mathrm{a}}, R_{\mathrm{a}}\right)-(+)-\mathrm{Cu}-4 \mathrm{a}$

b) Pd-catalyzed Asymmetric Intramolecular $\alpha$-Arylation (AIA)

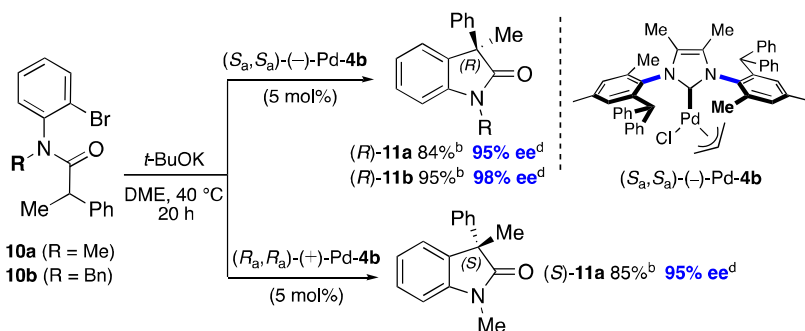

${ }^{a}$ Molar ratio of $\gamma / \alpha$ adduct were monitored by ${ }^{1} \mathrm{H}$ NMR spectroscopy analysis. ${ }^{b}$ Isolated yields after $\mathrm{SiO}_{2}$ chromatography. ${ }^{c}$ Determined by chiral-phase GC analysis. ${ }^{d}$ Determined by chiral-phase HPLC analysis. 
From the copper-catalysts library ${ }^{19}$, optically pure $C_{l^{-}}$ symmetric $\left(S_{\mathrm{a}}\right)-(+)-\mathrm{Cu}-3 \mathrm{~h}$ and $C_{2}$-symmetric $\left(R_{\mathrm{a},} R_{\mathrm{a}}\right)-(+)-$ $\mathrm{Cu}-4$ a featuring respectively ortho- $\mathrm{F} / \mathrm{CHPh}_{2}$ and ortho$\mathrm{H} / \mathrm{CHPh}_{2}$ substituents were found to be the best catalysts. The desired $\gamma$-adducts 9a,b were produced with complete regioselectivity and up to $90 \%$ ee, and isolated in excellent yields. ${ }^{20}$ Advantageously, by using opposite enantiomers of $\mathrm{Cu}$-complexes, the $\gamma$-adducts (ent)-9b could also be produced with similar efficiencies. The second asymmetric transformation investigated was the asymmetric intramolecular $\alpha$-arylation ${ }^{21}$ of amides $\mathbf{1 0}$ (Scheme 5,b). Among $C_{2}$-symmetric Pd-complexes ${ }^{22}$, the homochiral $\left(S_{\mathrm{a}}, S_{\mathrm{a}}\right)-(-)-\mathrm{Pd}-\mathbf{4} \mathbf{b}$ afforded the highest enantioselectivity. Resulting adducts $(R)-\mathbf{1 1} \mathbf{a}, \mathbf{b}$ were isolated in good to excellent yields and remarkable chiral inductions (95 to $98 \%$ ee). Of note, $(S)$-11a was also obtained in similar good yield and ee using $\left(R_{\mathrm{a}}, R_{\mathrm{a}}\right)-(+)-$ Pd-4b.

In summary, a novel access to chiral $C_{1}$ - and $C_{2}$ symmetric NHC-TM complexes from readily accessible prochiral NHC precursors was developed. As predicted by DFT calculations, the appropriate choice of the $\mathrm{N}$ aryl ortho-substituents and the NHC backbone substituents induced an axis of chirality which is constrained by the carbene-metal coordination. Resulting configurationally stable atropisomers of TM-complexes were successfully separated by preparative chiral HPLC in good yields and up to $>99.5 \%$ ee. For the first time, an optically pure Cu-complex was successfully transmetaled to gold and palladium counterparts in excellent yields with a full stereoretentivity $(>99.5 \%$ ee). Valuable insights to the transmetalation pathway were therefore obtained, supporting the hypothesis of an associative mechanism. The catalytic performances of these $\mathrm{Cu}$ - and Pd-complexes were illustrated in $\mathrm{Cu}-\mathrm{AAA}$ and Pd-AIA with up to $98 \%$ ee.

\section{ASSOCIATED CONTENT}

\section{Supporting Information}

The Supporting Information is available free of charge on the website.

NMR spectra of products, HPLC traces, experimental procedures and single crystal X-ray diffraction (PDF)

$\mathrm{X}$-ray crystallographic data (CIF)

\section{AUTHOR INFORMATION}

\section{Corresponding Authors}

*E-mail: herve.clavier@univ.amu.fr

*E-mail:marc.mauduit@ensc-rennes.fr

\section{ORCID}

Lingyu Kong: 0000-0002-7125-680X

Jennifer Morvan: 0000-0003-1210-7975

Delphine Pichon: 0000-0002-2307-6388

Marion Jean: 0000-0003-0524-8825

Muriel Albalat: 0000-0002-0215-0999

Thomas Vives: 0000-0001-6533-0703

Sophie Colombel-Rouen: 0000-0003-2603-2783

Michel Giorgi: 0000-0002-4367-1985
Vincent Dorcet: 0000-0001-9423-995X

Thierry Roisnel: 0000-0002-6088-4472

Christophe Crévisy: 0000-0001-5145-1600

Didier Nuel: 0000-0002-5055-0583

Paola Nava: 0000-0002-8909-8002

Stéphane Humbel : 0000-0001-5405-1848

Nicolas Vanthuyne: 0000-0003-2598-7940

Marc Mauduit : 0000-0002-7080-9708

Hervé Clavier: 0000-0002-2458-3015

Notes

The authors declare no competing financial interests.

\section{ACKNOWLEDGMENT}

We are grateful to the CNRS, Aix-Marseille Université, the ECM and the ENSCR. L.K. thanks the China Scholarship Council for a Ph.D. grant. This work was supported by the Region Bretagne (ARED 2018 "Biometa" N 601, grant to J.M.). M.M and D.P. thank the Agence Nationale de la Recherche (ANR-16-CE07-0019 Hel-NHC). Umicore AG $\&$ Co is acknowledged for a generous gift of complexes.

\section{REFERENCES}

(1) Selected books or reviews on NHCs, see: (a) N-Heterocyclic Carbenes: From Laboratory Curiosities to Efficient Synthetic Tools (Eds.: S. Díez-González), RSC Catalysis series, RSC Publishing: Cambridge, 2011; (b) Hopkinson, M. N.; Richter, C.; Schedler, M.; Glorius, F. An Overview of N-Heterocyclic Carbenes. Nature 2014, 510, 485-496; (c) Science of Synthesis: N-Heterocyclic Carbenes in Catalytic Organic Synthesis; Nolan, S. P., Cazin, C. S. J., Eds.; Thieme: Stuttgart, 2016; Vols. 1 and 2; For a review dealing with NHC TM complexes in catalysis, see: (d) Díez-Gonzalez, S.; Marion, N.; Nolan, S. P. N-Heterocyclic Carbenes in Late Transition Metal Catalysis. Chem. Rev. 2009, 109, 3612-3676.

(2) Powell, M. T.; Hou, D.-R.; Perry, M. C.; Cui, X.; Burgess, K. Chiral Imidazolylidine Ligands for Asymmetric Hydrogenation of Aryl Alkenes. J. Am. Chem. Soc. 2001, 123, 8878-8879

(3) Selected reviews on chiral NHC in catalysis: (a) Wang, F.; Liu, L.-J. Wang, W.; Li, S.; Shi, M. Chiral NHC-metal-based asymmetric catalysis. Coord. Chem. Rev. 2012, 256, 804-853. (b) Janssen-Müller, D.; Schlepphorst, C.; Glorius, F. Privileged Chiral N-Heterocyclic Carbene Ligands for Asymmetric Transition-Metal Catalysis. Chem. Soc. Rev., 2017, 46, 4845-4854.

(4) For a seminal study on the chiral relay, see: Luan, X.; Mariz, R.; Robert, C.; Gatti, M.; Blumentritt, Linden, A.; Dorta, R. Matching the Chirality of Monodentate N-Heterocyclic Carbene Ligands: A Case Study of Well-Defined Palladium Complexes for the Asymmetric $\alpha$-Arylation of Amides. Org. Lett. 2008, 10, 5569-5572.

(5) The synthesis of chiral-at-complexes from achiral NHC ligand precursors was recently reported, see: Hong, Y.; Jarrige, L.; Harms, K.; Meggers, E. Chiral-at-Iron Catalyst: Expanding the Chemical Space for Asymmetric Earth-Abundant Metal Catalysis. J. Am. Chem. Soc. 2019, 141, 4569-4572.

(6) The chiral HPLC resolution of chiral transition-metal complexes was scarcely reported, and to the best of our knowledge, none of them was used as chiral catalyst, see: (a) Norel, L.; Rudolph, M.; Vanthuyne, N.; Williams, J. A. G.; Lescop, C.; Roussel, C.; Autschbach, J.; Crassous, J.; R. Réau, R. Metallahelicenes: Easily Accessible Helicene Derivatives with Large and Tunable Chiroptical Properties. Angew. Chem. Int. Ed. 2010, 49, 99-102; (b) Hellou, N.; JahierDiallo, C.; Baslé, O.; Srebro-Hooper, M.; Toupet, L.; Roisnel, T.; Caytan, E.; Roussel, C.; Vanthuyne, N.; Autschbach, J.; Mauduit, M.; Crassous, J. Electronic and Chiroptical Properties of Chiral Cycloiridiated Complexes Bearing Helicenic NHC Ligands. Chem. Commun. 2016, 52, 9243-9246; (c) Hellou, N.; Srebro-Hooper, M.; Favereau, L.; Zinna, F.; Caytan, E.; Toupet, L.; Dorcet, V.; Jean, M.; Vanthuyne, N.; Williams, J. A. G.; Di Bari, L.; Autschbach, J.; Crassous, J. Enantiopure Cycloiridiated Complexes Bearing a Pentahelicenic N- 
Heterocyclic Carbene and Displaying Long-Lived Circularly Polarized Phosphorescence Angew. Chem. Int. Ed. 2017, 56, 8236-8239.

(7) (a) Ōki, M. Recent Advances in Atropisomerism, in Topics in Stereochemistry, Vol. 14 (Eds.: Allinger, N. L.; Eliel, E. L;. Wilen, S. H.), Hoboken, NJ, John Wiley \& Sons, 1983, pp. 1-82; (b) Ibon, A.; Elguero, J.; Roussel, C.; Vanthuyne, N.; Piras, P. Atropisomerism and Axial Chirality, in Heteroaromatic Compounds. Advances in Heterocyclic Chemistry, 2012, 105, 1-188.

(8) (a) Adamo, C.; Barone, V. Toward Reliable Density Functional Methods without Adjustable Parameters: The PBE0 Model. J. Chem. Phys. 1999, 110, 6158-6170; (b) Weigend, F.; Ahlrichs, R. Balanced Basis Sets of Split Valence, Triple Zeta Valence and Quadruple Zeta Valence Quality for $\mathrm{H}$ to Rn: Design and Assessment of Accuracy. Phys. Chem. Chem. Phys. 2005, 7, 3297-3305; (c) Grimme, S.; Antony, J.; Ehrlich, S.; Krieg, H. A Consistent and Accurate Ab Initio Parametrization of Density Functional Dispersion Correction (DFTD) for the 94 Elements H-Pu. J. Chem. Phys. 2010, 132, 154104154104; (d) Frisch, M.; Trucks, G.; Schlegel, H.; Scuseria, G.; Robb, M.; Cheeseman, J.; Scalmani, G.; Barone, V.; Mennucci, B.; Petersson, G.; et al. Gaussian 09, Revision D.01; Gaussian, Inc., Wallingford CT, 2013.

(9) Imidazolium salts were prepared in moderate yields by adapting protocols from the literature, see: (a) Zhang, J.; Fu, J.; Su, X.; Wang, X.; Song, S.; Shi, M. Synthesis of Various Saturated and Unsaturated N-Heterocyclic Carbene Precursors by Triflic Anhydride Mediated Intramolecular Cyclization. Chem. Asian J. 2013, 8, 552-555;

(10) For the synthesis of $\mathrm{Cu}-\mathrm{NHC}$ complexes, see for instance: Lazreg, F.; Cazin, C. S. J. NHC-copper complexes and their applications in N-Heterocyclic Carbenes - Effective Tools for Organometallic Synthesis; Nolan, S. P. Ed.; Wiley-VCH, Mannheim, 2014, 199242.

(11) Wendt, O. F. Transmetalation Reactions Involving Group 10 Metals. Curr. Org. Chem. 2007, 11, 1417-1433;

(12) For previous works on transmetalation with coppercomplexes, see: (a) Santoro, O.; Lazreg, F.; Cordes, D. B.; Slawin, A. M. Z.; Cazin, C. S. J. Homoleptic and Heteroleptic Bis-NHC Cu(I) Complexes as Carbene Transfer Reagents. Dalton Trans. 2016, 45, 4970-4973; (b) Nahra, F.; Gomez-Herrera, A.; Cazin, C. S. J. Copper(I)-NHC Complexes as NHC Transfer Agents. Dalton Trans. 2017, 46, 628-631.

(13) For selected recent examples of synthesis of Pd- and Au-NHC complexes, see: (a) Vanden Broeck, S. M. P.; Nahra, F.; Cazin, C. S. J. Bulky-yet-flexible Carbene Ligands and Their Use in Palladium Cross-coupling. Inorganics, 2019, 7, 78; (b) Zinser, C. M.; Nahra, F.; Falivene, L.; Brill, M.; Cordes, D. B.; Slawin, A. M. Z.; Cavallo, L.; Cazin, C. S. J.; Nolan, S. P. Synthesis and Reactivity of $[\mathrm{Au}(\mathrm{NHC})$ (Bpin)] Complexes. Chem. Commun. 2019, 55, 6799-6802.

(14) See Supporting Information, Table S1, entry 5.

(15) These values are in line with those determined both by DFT calculations and experimentally in previous reports, see: (a) Luan, X.; Mariz, R.; Gatti, M.; Costabile, C.; Poater, A.; Cavallo, L.; Linden, A.; Dorta, R. Identification and Characterization of a New Family of Catalytically Highly Active Imidazolin-2-ylidenes. J. Am. Chem. Soc. 2008, 130, 6848-6858; (b) Laidlaw, G.; Wood, S. H.; Kennedy, A. R.; Nelson, D. J. An N-Heterocyclic Carbene with a Saturated Backbone and Spatially-Defined Steric Impact. Z. Anorg. Allg. Chem. 2019, 645, 105-112.

(16) See Supporting Information, Scheme SX and SX.

(17) See Supporting Information, Scheme S9.

(18) Selected book chapter or review on Cu-AAA, see: (a) Baslé, O.; Denicourt-Nowicki, A.; Crévisy, C.; Mauduit, M. Asymmetric Allylic Alkylation in Copper-Catalyzed Asymmetric Synthesis (Eds: A. Alexakis, N. Krause, S. Woodward) Wiley, 2014, Chapter 4, pp. 85-125. (b) Alexakis, A.; Backvall, J. E.; Krause, N.; Pamies, O.; Dieguez, M. Enantioselective Copper-Catalyzed Conjugate Addition and Allylic Substitution Reactions. Chem. Rev. 2008, 108, 2796-2823.

(19) See Supporting Information, Table S22.

(20) It is noteworthy that the chiral induction reached here is one of the best reported so far regarding the class of $C_{l}$-symmetric monodentate NHCs, see ref. 3.
(21) For a selected example of successful Pd-AIA promoted by chiral NHC ligands, see: Kündig, E.P.; Seidel, T.M.; Jia, Y.-X.; Bernardinelli, G. Bulky Chiral Carbene Ligands and Their Application in the Palladium-Catalyzed Asymmetric Intramolecular $\alpha$-Arylation of Amides. Angew. Chem. Int. Ed. 2007, 46, 8484-8487. See also ref. 4.

(22) See Supporting Information, Table S24. 


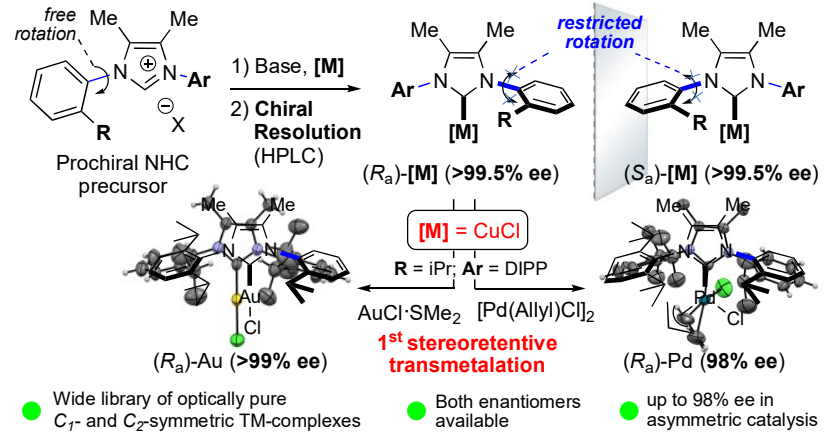

